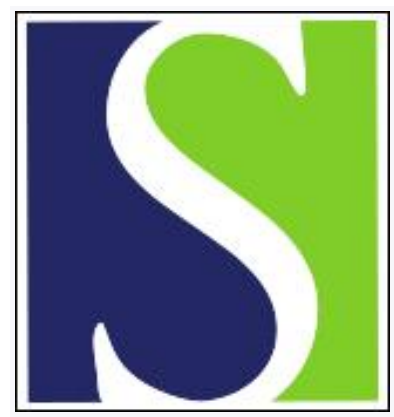

Scand J Work Environ Health 2017;43(1):86-94

https://doi.org/10.5271/sjweh.3602

Published online: 09 Nov 2016, Issue date: 01 Jan 2017

Association between high ambient temperature and acute work-related injury: a case-crossover analysis using workers' compensation claims data

by McInnes JA, Akram M, MacFarlane EM, Keegel T, Sim MR, Smith P

This study found that high ambient temperatures are associated with increased risk of serious acute work-related injury for some worker subgroups. It expanded on previous research by assessing associations with both daily minimum and maximum temperatures, by using a novel approach to occupational characteristics classification, and by using a case-crossover study design. Study findings provide evidence to inform prevention strategies.

Affiliation: Monash Centre for Occupational and Environmental Health (MonCOEH), Department of Epidemiology and Preventive Medicine, Monash University, Level 5, the Alfred Centre, 99 Commercial Road, Melbourne Victoria, 3004, Australia. Judy.mcinnes@monash.edu

The following articles refer to this text: 2019;45(2):194-202; 2019;45(6):588-599; 2020;46(1):1-4

Key terms: ambient temperature; analysis; case crossover study; case-crossover study; climate change; compensation claim; occupational health; occupational health; temperature; work injury; work-related injury; workers' compensation claims data

This article in PubMed: www.ncbi.nlm.nih.gov/pubmed/27829252

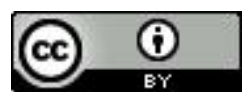




\title{
Association between high ambient temperature and acute work-related injury: a case-crossover analysis using workers' compensation claims data
}

\author{
by Judith A McInnes, MPH, ${ }^{1}$ Muhammad Akram, PhD, ${ }^{1}$ Ewan M MacFarlane, PhD, ${ }^{1}$ Tessa Keegel, PhD, ${ }^{1,2}$ \\ Malcolm R Sim, PhD, ${ }^{1}$ Peter Smith, PhD, ${ }^{1,3}$
}

\begin{abstract}
McInnes JA, Akram M, MacFarlane EM, Keegel T, Sim MR, Smith P. Association between high ambient temperature and acute work-related injury: a case-crossover analysis using workers' compensation claims data. Scand J Work Environ Health. 2017;43(1):86-94. doi:10.5271/sjweh.3602
\end{abstract}

\begin{abstract}
Objectives The aim of this study was to investigate the association between high ambient temperature and acute work-related injury, expanding on previous research in this area. Specifically we examined the relationship between both daytime and overnight temperatures and injury risk and disentangled physically demanding occupational exposures from exposure to outdoor working conditions.

Methods A time-stratified case-crossover study design was used to examine the association between ambient temperatures and acute work-related injuries in Melbourne, Australia, 2002-2012, using workers' compensation claims to identify work-related injuries. The relationship was assessed for both daily maximum and daily minimum temperatures using conditional logistic regression.

Results Significant positive associations between temperature and acute work-related injury were seen for younger workers ( $<25$ years), with the odds of injury increasing by $1 \%$ for each $1{ }^{\circ} \mathrm{C}$ increase in daily minimum temperature, and by $0.8 \%$ for each $1{ }^{\circ} \mathrm{C}$ increase in daily maximum temperature. Statistically significant associations were also observed between daily maximum temperature and risk of injury for workers employed in the highest strength occupations and for male workers, and between daily minimum temperature and injury for all cases combined, female workers, workers aged 25-35 and $\geq 55$ years, "light" and "limited" physical demand groups, and "in vehicle or cab" and "regulated indoor climate" workplace exposure groups.
\end{abstract}

Conclusions Young workers, male workers and workers engaged in heavy physical work are at increased risk of injury on hot days, and a wider range of worker subgroups are vulnerable to injury following a warm night. In light of climate change projections, this information is important for informing injury prevention strategies.

Key terms case crossover study; climate change; occupational health; work injury.

In addition to contributing to a vast global health and economic burden, injuries acquired during the course of employment exact a heavy toll on individual workers, their co-workers and families. The International Labor Organization estimates that world-wide, 317 million workplace accidents occur each year, equating to 153 accidents every 15 seconds (1). Economic costs of work-related health problems including injuries are estimated to equal $4-6 \%$ of the gross domestic product for most countries (2). Identifying and assessing risk factors for work-related injury is essential for informing evidence-based injury prevention policies, programs and practices.

There is some evidence that hot weather is a risk factor for acute work-related injury (3-6). Understanding any association between ambient heat and workrelated injury is particularly important in the context of a warming climate. Average global surface temperatures have increased by $0.85^{\circ} \mathrm{C}$ since 1880 and, for all greenhouse gas emission scenarios, are projected to continue

1 Monash Centre for Occupational and Environmental Health (MonCOEH), Department of Epidemiology and Preventive Medicine, Monash University, Melbourne Victoria, Australia.

2 School of Psychology and Public Health, La Trobe University, Bundoora, Victoria, Australia.

3 Institute for Work \& Health, Toronto, Canada.

Correspondence to: Judith A McInnes, Monash Centre for Occupational and Environmental Health (MonCOEH), Department of Epidemiology and Preventive Medicine, Monash University, Level 5, the Alfred Centre, 99 Commercial Road, Melbourne Victoria, 3004, Australia. [E-mail: Judy.mcinnes@monash.edu] 
increasing over future decades (7). This warming will be experienced as more hot days and warm nights, fewer cool days and cold nights, and more frequent, hotter and longer heatwaves $(8,9)$. While the impact of hot weather and heatwaves on mortality and morbidity have been well documented at the population level $(10,11)$, characterization of the impact of hot weather on occupational health is a relatively neglected area. The very few studies that have focused on the relationship between high ambient temperature and work-related injury are difficult to compare as they differ in exposure measures and outcome definitions and provide some contradictory findings (3-6).

Particular segments of the labor market may be susceptible to the effects of heat on work injury risk. For example, three studies $(3,5,6)$ have identified young workers as being at increased risk of injury, of which two $(5,6)$ identified male workers as a vulnerable group. The relationship between occupation and risk of acute work-related injury during hot weather is less clear, with some studies finding a stronger relationship between increased temperature and risk of injury among "outdoor" workers (5), than others (6). These inconsistent findings may be due to the classification systems used, with previous studies often inferring particular working conditions for all workers in a given industry (eg, construction) when this is likely not the case.

To date, most studies have assessed the association between daytime thermal conditions and work-related injury (4-6). There has been less focus on overnight minimum temperature prior to the day of injury despite the potential impact of warm overnight temperatures on sleep quality and subsequent fatigue at work.

Finally, not all previous studies have taken into account the potential confounding of the relationship between increased temperature and risk of work injury by demographic and labor market factors that may vary by year, month, or day of the week (eg, for certain occupations working hours tend to be greater towards the end of the week and over the weekend) (12). By using a case-crossover study design, considered an ideal tool for examination of associations between short-term exposures and acute health outcomes, these confounders, individual level time-stable confounders, and longer term time trends can be controlled by design rather than through complex modelling required with time-series analyses $(13,14)$.

The objective of this study was to investigate the association between high ambient temperature and acute work-related injury, expanding on the small body of previous research in this area. Specifically, we examine the relationship between both daytime and overnight temperature and injury risk and disentangle physically demanding occupational exposures from exposure to outdoor working conditions.

\section{Method}

\section{Approach and setting}

Using a case-crossover study design and workers' compensation claims to identify work-related injury events, we examined the association between outdoor summer temperatures and acute work-related injuries sustained by workers in the metropolitan Melbourne area, Australia, from November to March in 2002-2012.

Melbourne, the capital city of the south eastern Australian state of Victoria, has a population of approximately 4.5 million (as of June 2015) (15). It has a temperate climate characterized by changeable weather conditions and warm-to-hot summers $(16,17)$. The Melbourne metropolitan area, which includes the city of Melbourne and surrounding suburbs, consists of 31 local government areas (LGA), the outer borders of which geographically define the area.

\section{Outcome data}

Administrative data derived from accepted workers' compensation claims submitted to WorkSafe Victoria, the statutory authority responsible for managing the Victorian workers' compensation scheme, was obtained from the compensation research database for the financial years 2002-2012 (18). Approximately 85\% of Victorian workers are covered under the WorkCover Victoria workers' compensation scheme, with the remainder being predominantly self-employed workers or those employed by self-insured employers or by the Australian Commonwealth Government which has its own insurance scheme (19). Work-related injuries are eligible for WorkSafe Victoria compensation if they result in $\geq 10$ days absence from work or incur healthcare costs above a threshold value, but not if they are sustained on the journey to or from work (20).

Information collected with each compensation claim included information about the injured worker (age, gender), characteristics of their employment (occupation and industry), and their injury (date, work place, nature of injury). To maintain confidentiality, data was provided in a de-identified form and had been previously aggregated to the 31 LGA of the Melbourne metropolitan area using postal codes. Information about each claim included the worker's residential LGA and the LGA of the workplace where the injury occurred.

For this study, a case was defined as an accepted worker's compensation claim for a non-fatal or fatal acute injury to a worker aged $\geq 15$ years, inflicted at a workplace located in the Melbourne metropolitan area during the warmest months of the year [November to March (inclusive)], during the period 1/11/2002$31 / 12 / 2012$. Our initial sample of workers' compen- 
sation claims that met these inclusion criteria totaled 49321 claims. From this sample, we removed claims for which meteorological data were missing $(\mathrm{N}=1292)$ and for which the injury occurred on a public holiday as these days were likely to have potentially confounding characteristics that were unlike other workdays $(\mathrm{N}=1089)$. This left a final sample of 46940 claims for analysis ( $95 \%$ of the original sample).

\section{Meteorological data}

Historical meteorological data recorded by 16 weather stations located within the Melbourne metropolitan area over the period 2002-2012 was obtained from the Australian Bureau of Meteorology. Of these, only 8 had recorded temperature data over the entire study period. Data from these weather stations was allocated to the 31 LGA on the basis of proximity to the most populous part of that LGA (or the center of densely populated inner-city LGA). Each LGA was allocated data from one weather station only.

For our analysis, we focused on two measures of temperature: maximum daily temperature (maximum temperature in the 24 hours after 09:00 hours), and minimum daily temperature (minimum temperature in the 24 hours before 09:00 hours). In Melbourne, daily maximum and minimum temperatures are typically reached between 14:00-17:00 hours and 05:00-06:00 hours, respectively $(21,22)$. Therefore maximum daily temperature was used as a measure of the maximum daytime temperature during the day of injury, while minimum daily temperature was used to measure the minimum temperature during the night before the day of injury. We also extracted values for relative humidity as this influences the effectiveness of thermoregulation (23). To most closely match relative humidity to maximum and minimum temperatures, we used the relative humidity data recorded at 15:00 and 06:00 hours, respectively.

\section{Occupational characteristics}

Each compensation claim included the claimant's occupation coded according to the Australian and New Zealand Standard Classification of Occupations (ANZSCO) system. Using these codes, we grouped occupations based on their physical demands and their potential workplace temperature exposures. The classification system used to assign particular strength and potential workplace temperature exposures was originally developed by Human Resources and Skills Development Canada (HRSDC) to assign particular characteristics to occupations (24). Trained occupational analysts assigned these characteristics to each occupational code using a modified Delphi procedure (24). The relationship between Canadian occupational codes and the ANZSCO codes was derived using a common coding structure for each system: the International Standard Classification of Occupations (ISCO - 88) (International Labor Office, 1988). The validity of this system has been previously demonstrated using workers' compensation data in Victoria $(25,26)$.

Physical demands (based on minimum strength requirements) were classified into four handling load categories: (i) limited ( $\leq 5 \mathrm{~kg}$ ), (ii) light $(5-10 \mathrm{~kg}$ ), (iii) medium (10-20 kg), and (iv) heavy ( $>20 \mathrm{~kg})$. Potential workplace temperature exposures were classified into five groups: (i) working only in a vehicle or cab; (ii) outdoors with no work in a regulated indoor climate; (iii) regulated indoor climate; (iv) unregulated indoor climate and vehicle or cab; and (v) indoor and outdoor work.

\section{Design and analysis}

The association between ambient outdoor temperatures and work-related injury was investigated using a timestratified case-crossover study design (13), whereby, for each case, temperature exposure on the date of injury affliction (case day) was compared to exposures when injury did not occur [on the same day of the week, for other weeks in the same calendar month and year (referent days)], using conditional logistic regression analysis models. Using this approach, known and unknown time-invariant individual level confounders (eg, age, gender, fitness, training, experience) are controlled by design through cases serving as their own controls. By matching case and referent days by day of the week, month, and year, these confounders are also controlled through design. As referent days are selected within a short period of time from case days, confounding by seasonal effects and long-term trends is minimized. We ran separate conditional logistic regression models for daily maximum and minimum temperature exposures. The study was restricted to the months November to March (inclusive) as these are the five warmest months in Melbourne. Maximum daily temperatures were assigned to case and referent days according to the LGA of each claimant's workplace, while minimum daily temperatures were assigned to each case according to the claimant's residential LGA. Maximum temperature models were adjusted for workplace LGA relative humidity at 15:00 hours, and minimum temperature models were adjusted for residential LGA relative humidity at 06:00 hours.

In light of previous studies reporting non-linear heat-injury relationships (3-5), we initially assessed the relationship between temperature and the risk of work-related injury for linearity by including a squared temperature term in the maximum and minimum temperature models. When the squared term indicated a curvilinear relationship, we subsequently estimated the predicted probabilities based on the regression coef- 
ficients (for temperature and temperature squared) to better understand the shape of the relationship between temperature and injury. Where the squared term was not significant, based on inspection of $95 \%$ confidence intervals $(95 \% \mathrm{CI})$ and P-values, we removed it from the model and estimated the relationship between linear temperature increase and risk of injury.

Given that previous studies have found the relationship between temperature and injury differs for particular labor market subgroups, we conducted a further series of separate models for each temperature metric stratified by gender (male/female), age group (15-24, 25-34, 35-44, 45-54 and $\geq 55$ years), and occupational characteristics. In this way, each subgroup analysis had its own model and goodness-of-fit estimate (using the likelihood ratio test).

Results are presented as odds ratios (OR) and 95\% CI of acute work-related injury associated with a $1{ }^{\circ} \mathrm{C}$ increase in temperature measure. All analyses were conducted using Stata V.12 (Stata/IC 12.1.2011, StataCorp, College Station LP, Texas, USA). P-values of $<0.05$ were considered statistically significant.

\section{Ethics}

On the basis that only non-identifiable data was to be used in this study, exemption from ethics review was received from the Monash University Human Research Ethics Committee on 13 December, 2012.

\section{Results}

Characteristics of the 46940 accepted compensation claims that satisfied our case definition are provided in table 1 . The injury date for each of these claims defined the case day. The majority of claimants were male (72\%) and aged $34-54$ years (49\%). After grouping occupations according to occupational characteristics, the physical demand groups most frequently represented were "limited" and "light" (62\% when combined) and for potential workplace temperature exposures this was "temperature regulated indoor climate" (45\%).

Using the time-stratified approach to referent day selection previously described, the 46940 case days were matched with a total of 147864 referent days (excluding public holidays and days with missing meteorological data). In this way, each case day was matched with up to 4 referent days: $58.6 \%$ of cases were matched with 3 referent days and $28.5 \%$ were matched with 4 referent days.

Table 2 shows that over the study period average ambient temperatures derived from data recorded by eight weather observation stations located in the Mel-
Table 1. Number and characteristics of accepted workers' compensation claims for acute work-related injury, metropolitan Melbourne, 2002-2012 (November-March).

\begin{tabular}{lrr}
\hline & Number & $\%$ \\
\cline { 2 - 3 } Total Melbourne metropolitan area & 46940 & 100.0 \\
Gender & & \\
Male & 33651 & 71.7 \\
Female & 13289 & 28.3 \\
Age group (years) & & \\
$<25$ & 6241 & 13.3 \\
$25-34$ & 9768 & 20.8 \\
$35-44$ & 11473 & 24.4 \\
$45-54$ & 11729 & 25.0 \\
$\geq 55$ & 7729 & 16.5 \\
Physical demands (minimum handling load) & & \\
Limited ( $\leq 5 \mathrm{~kg})$ & 14285 & 31.1 \\
Light (5-10 kg) & 14586 & 31.1 \\
Medium (10-20 kg) & 12836 & 27.3 \\
$\quad$ Heavy (>20 kg) & 5233 & 11.1 \\
Potential workplace temperature exposures & & \\
$\quad$ Working only in a vehicle or cab & 3720 & 7.9 \\
Outdoors with no work in regulated indoor climate & 2078 & 4.4 \\
Regulated indoor climate & 21244 & 45.3 \\
$\quad$ Unregulated indoor climate and vehicle or cab & 3363 & 7.2 \\
Indoor and outdoor work & 16535 & 35.2 \\
\hline
\end{tabular}

bourne metropolitan area ranged from a daily maximum temperature of $10.5-47.5^{\circ} \mathrm{C}$ and daily minimum temperature of $0.2-30.5^{\circ} \mathrm{C}$.

\section{Assessment of linearity}

There was no evidence of non-linearity in the relationship between maximum daily temperature and risk of work injury. For minimum daily temperature, evidence of a non-linear relationship between temperature and risk of injury was present for all cases combined and for various subgroups, namely, (i) female workers, (ii) age groups $25-35$ and $\geq 55$ years, (iii) light and limited physical demand groups, and (iv) "working only in a vehicle or cab" and "regulated indoor only" workplace exposure groups.

Figures 1 and 2 show changes in the predicted probabilities derived from regression coefficients for temperature and temperature squared, for minimum temperature values between $1.0-30.5^{\circ} \mathrm{C}$. All curves show a U-shaped relationship, with risk of injury declining with increasing temperature up to temperature values of approximately $12-14{ }^{\circ} \mathrm{C}$ and risk of injury increasing with increasing temperature after this point.

Association between ambient temperature and acute work-related injury.

Table 3 presents the OR and 95\% CI for associations between ambient temperature and compensable acute work-related injury for all cases combined, demographic subgroups, and occupational characteristic subgroups. For maximum daily temperature, estimates presented are 
Table 2. Descriptive summary of daily meteorological data, metropolitan Melbourne, Victoria, 2002-2012 (November-March). [IQR=Interquartile range]

\begin{tabular}{|c|c|c|c|c|c|c|c|}
\hline \multirow[t]{2}{*}{ Meteorological measure (unit) } & \multirow[t]{2}{*}{ Minimum } & \multirow[t]{2}{*}{ Maximum } & \multirow[t]{2}{*}{ Mean } & \multicolumn{4}{|c|}{ Percentiles } \\
\hline & & & & $25 \%$ & $50 \%$ & $75 \%$ & $\mathrm{IQR}^{\mathrm{a}}$ \\
\hline Maximum daily temperature $\left({ }^{\circ} \mathrm{C}\right)$ & 10.5 & 47.5 & 25.1 & 20.8 & 24 & 28.8 & 8 \\
\hline Minimum daily temperature $\left({ }^{\circ} \mathrm{C}\right)$ & 0.2 & 30.5 & 13.5 & 11.2 & 13.4 & 15.7 & 4.5 \\
\hline Relative humidity at 15.00 hours (\%) & 3 & 100 & 48.8 & 38 & 49 & 59 & 21 \\
\hline Relative humidity at 06.00 hours (\%) & 1 & 100 & 78.9 & 71 & 82 & 90 & 19 \\
\hline
\end{tabular}

a Difference between the $25^{\text {th }}$ and $75^{\text {th }}$ percentiles of the measured parameter.

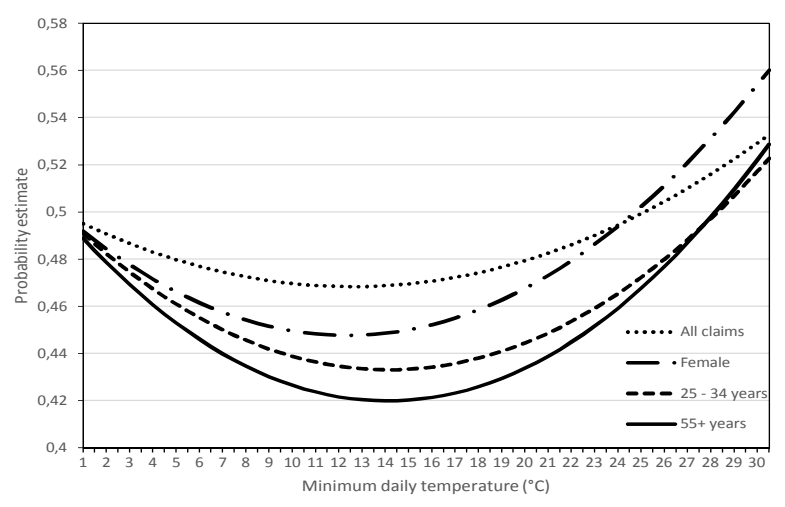

Figure 1. Curvilinear relationships between minimum daily temperature, all claims and subgroups defined by gender, and age group.

those of conditional logistic regression models assuming linear temperature - injury relationships. As both linear and curvilinear relationships between minimum daily temperature and acute work-related injury were identified, estimates of association using models assuming linearity, and also of models for which evidence of a curvilinear relationship was evident on incorporation of a squared minimum daily temperature term, have been presented for this temperature measure in two separate columns of table 3 .

Overall the strongest associations were seen between both temperature measures and acute work-related injury of younger workers ( $<25$ years), with the odds of injury increasing by $1 \%$ for each $1{ }^{\circ} \mathrm{C}$ increase in daily minimum temperature and by $0.8 \%$ for each $1{ }^{\circ} \mathrm{C}$ increase in daily maximum temperature. Statistically significant associations between daily maximum temperature and risk of injury were also observed for workers employed in the highest strength occupations and for male workers, and between daily minimum temperature and injury for all cases combined, female workers, workers aged 25-35 and $\geq 55$ years, light and limited physical demand groups, and "in vehicle or cab" and "regulated indoor climate" workplace exposure groups.

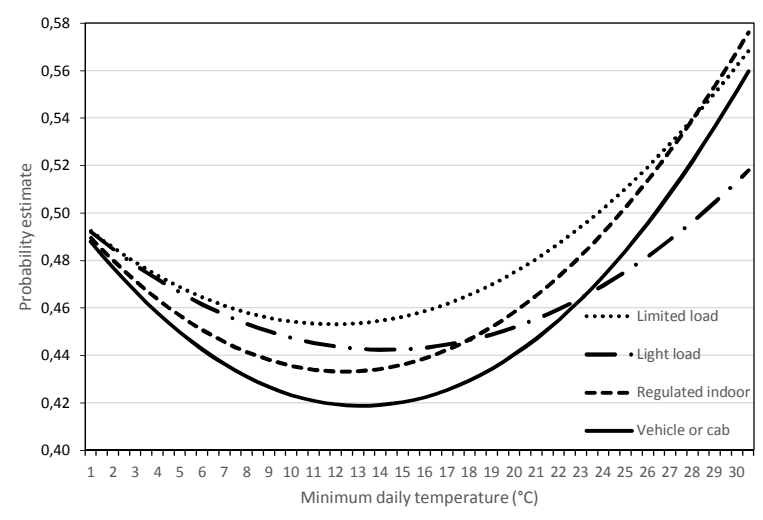

Figure 2. Curvilinear relationships between minimum daily temperature, and subgroups defined by occupational characteristics.

\section{Discussion}

This study adds to the existing body of evidence that high ambient temperature is associated with risk of acute work-related injury for some worker sub-groups. It has expanded on previous research by (i) assessing associations with daily minimum temperature as well as daily maximum temperature, (ii) using case-level data in a case-crossover study design, and (iii) using a novel classification of occupational characteristics

We observed that young workers, male workers and workers engaged in the most physically demanding occupations were more vulnerable to injury with increasing daytime temperatures. We also observed that minimum overnight temperature influenced risk of injury the following day for young workers and for female workers, older workers, and workers engaged in occupations that had the least onerous handling load requirements and whose workplace temperature exposure was restricted to that experienced when being in a vehicle or cab or in a regulated indoor climate.

The strength of associations between injury risk and maximum daytime temperature that we identified for young workers (OR 1.008, 95\% CI 1.001-1.015) and male workers (OR 1.003, 95\% CI 1.000-1.006) were similar to those reported by previous similar studies. 
Adam-Poupart et al (6) reported a significantly increased risk of acute injury to workers aged $<25$ years [injury risk ratio (IRR) $1.008,95 \%$ CI $1.005-1.010]$ and male workers (IRR 1.003, 95\% CI 1.002-1.005) when data for all health regions of Quebec, Canada, were combined. Xiang et al (5) found a $0.5 \%$ increase in all accepted injury claims (IRR 1.005, 95\% CI 1.002-1.008) for each $1{ }^{\circ} \mathrm{C}$ in daily maximum temperature $<37.9{ }^{\circ} \mathrm{C}$ for young workers and a $0.4 \%$ increase (IRR 1.004; $(95 \%$ CI 1.002-1.006) per $1^{\circ} \mathrm{C}$ in daily maximum temperature $<37.7^{\circ} \mathrm{C}$ for male workers.

We found no evidence of non-linearity in the relationship between maximum daily temperature and risk of work-related injury. This is in agreement with the findings of Adam-Poupart et al (6), but contrasts with Xiang et al (5) and Morabito et al (27) who found that reverse u-shaped curves best described this relationship. Different populations and analysis approaches are likely underlying factors influencing these discrepancies.

Our findings regarding risk of injury and minimum temperature are new. We have not located any other studies in the literature that have focused on the association between minimum daily temperature and acute workrelated injury. This measure, defined in our study as the minimum temperature in the 24 hours before 09:00 hours reflects the minimum overnight temperature prior to the day of injury. We found a stronger association between temperature and risk of injury to younger workers for daily minimum temperature than for daily maximum temperature, suggesting that overnight temperatures may have a stronger influence on risk of acute injury than maximum temperature for this age group. It is possible that this effect is mediated through poor quality sleep. A number of studies have identified insufficient sleep as a risk factor for injury in an occupational setting $(28,29)$, and lack of alertness has been suggested as a possible mechanism for this association. A recent study found that sleep restriction reduced vigilance in the heat and is a risk factor for heat illness (30). Our observations of a curvilinear relationship between daily minimum temperature and risk of injury showing minimal risk between approximately $12-14{ }^{\circ} \mathrm{C}$ may suggest an optimal sleep temperature. Further investigation of mechanisms underlying the observed associations between risk of injury and warm overnight minimum temperatures are warranted.

Our assessment of association between maximum daily temperature and risk of injury according to occupational characteristics differed to previous studies. We identified an association between maximum daily temperature and the highest physical demand occupations (OR 1.008, 95\% CI 1.001-1.016) but not between maximum daily temperature and the potential temperature exposure category "outdoors with no work in a regulated indoor climate". This differs to previous studies that have stratified their analyses according to industrial sec- tor aggregated to be outdoors or indoors. Xiang et al (5) found an increased risk of injury for outdoor industries combined (IRR 1.005, 95\% CI 1.001-1.009), while Adam-Poupart et al (6) found an increased risk of injury to workers of six mostly outdoor work industrial sectors combined but not for each of these sectors individually (IRR 1.004, 95\% CI 1.001- 1.006). Occupational groups categorized as heavy in the physical demand group (minimum handling loads $>20 \mathrm{~kg}$ ) include bricklayers, plumbers, metal engineering process workers, police, paramedics and emergency service workers, not all of whom would be classified as outdoor workers. Possible mechanisms behind the observed association include increased metabolic heat generated with strenuous muscle activity and use of heavy, impermeable personal protective clothing. Our classification system has allowed for an exploration of risk associated with physical demand separate from outdoor heat exposures. Our findings suggest that being outdoors in itself may not be the only factor increasing risk to acute workrelated injury on hot days.

The mechanism underlying an association between high ambient temperature and acute work-related injury is likely to be a combination of factors. Decrements in performance of perceptual motor tasks have been reported when ambient temperatures are high, and symptoms of heat exhaustion including fatigue and dizziness could compromise workplace safety $(31,32)$. Sweaty, hands, hot metal railings and tools, and foggy safety glasses, combined with hurrying to finish tasks before the heat of the day, could conceivably lead to slipping, dropping tools, falls and errors of judgment. Workers may discard restrictive personal protective clothing when feeling uncomfortably hot; lack of sleep during hot nights, as discussed, could impact on worker fatigue and risk of injury on subsequent days.

Further research is needed to examine the likely complex interplay of injury mechanisms underpinning the observed increase in risk of younger workers to injury during hot weather. Possible contributing factors could include inexperience, inadequate training, workplace pressures, hydration behaviors, consumption of alcohol, quality of housing and attitudes to strenuous physical work on hot days. Further studies, particularly qualitative studies, are needed to assess these factors and others to inform prevention programs. A recent study has reported that hospital emergency department (ED) data captures a greater proportion of injuries to younger people than compensation claims, with the rate of ED presentations for acute injury being 3.5 times greater than the rate of compensation claims for the 15-19-yearold age group in Victoria (33). Further investigation of the association between temperature and work-related injury to young people using ED data may shed more light on the injury burden for this age group. 
Table 3. Odds ratios (OR) of acute work-related injury for each $1{ }^{\circ} \mathrm{C}$ increase in daily maximum temperature, daily minimum temperature, and daily minimum temperature squared, Melbourne, 2002-2012, (November - March). [95\% Cl=95\% confidence interval.]

\begin{tabular}{|c|c|c|c|c|c|c|c|}
\hline & \multirow[t]{2}{*}{ Observations a } & \multicolumn{2}{|c|}{$\begin{array}{l}\text { Maximum } \\
\text { temperature }\end{array}$} & \multicolumn{2}{|c|}{$\begin{array}{l}\text { Minimum } \\
\text { temperature }\end{array}$} & \multicolumn{2}{|c|}{$\begin{array}{l}\text { Minimum } \\
\text { temperature squared } \mathrm{c}\end{array}$} \\
\hline & & $\mathrm{OR}$ & $95 \% \mathrm{Cl}$ & $\mathrm{OR}$ & $95 \% \mathrm{Cl}$ & $\mathrm{OR}$ & $95 \% \mathrm{Cl}$ \\
\hline All cases & 194790 & 1.002 & $0.999-1.004$ & . & & 1.001 & $1.000-1.001$ \\
\hline \multicolumn{8}{|l|}{ Gender } \\
\hline Male & 139630 & 1.003 & $1.000-1.006$ & 1.001 & $0.997-1.005$ & & \\
\hline Female & 55160 & 0.999 & $0.994-1.005$ & . & & 1.001 & $1.000-1.002$ \\
\hline \multicolumn{8}{|l|}{ Age group (years) } \\
\hline$<25$ & 25867 & 1.008 & $1.001-1.015$ & 1.010 & $1.001-1.020$ & & \\
\hline $25-34$ & 40528 & 1.002 & $0.996-1.007$ & & & 1.001 & $1.000-1.003$ \\
\hline $35-44$ & 47617 & 1.004 & $0.999-1.009$ & 1.006 & $0.999-1.013$ & . & (1) \\
\hline $45-54$ & 48666 & 0.999 & $0.994-1.003$ & 0.998 & $0.991-1.005$ & & \\
\hline$\geq 55$ & 32112 & 0.998 & $0.987-1.002$ & . & & 1.002 & $1.000-1.002$ \\
\hline \multicolumn{8}{|l|}{ Physical demands (minimum handling load) } \\
\hline Limited $(\leq 5 \mathrm{~kg})$ & 59259 & 1.000 & $0.996-1.005$ & . & & 1.001 & $1.000-1.002$ \\
\hline Light $(5-10$ kg) & 60519 & 1.003 & $0.998-1.007$ & & & 1.001 & $1.000-1.002$ \\
\hline Medium $(10-20$ kg) & 53370 & 0.999 & $0.995-1.004$ & 0.999 & $0.992-1.006$ & . & . \\
\hline Heavy $(>20$ kg) & 21642 & 1.008 & $1.001-1.016$ & 1.009 & $0.998-1.020$ &  & 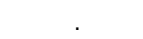 \\
\hline \multicolumn{8}{|l|}{ Potential workplace temperature exposures } \\
\hline Working only in a vehicle or cab & 15461 & 1.008 & $0.999-1.016$ & & & 1.002 & $1.000-1.004$ \\
\hline Outdoors, no work in regulated indoor climate & 8631 & 0.997 & $0.986-1.009$ & 0.994 & $0.978-1.011$ & & \\
\hline Regulated indoor climate & 88251 & 1.002 & $0.999-1.006$ & 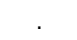 & & 1.002 & $1.001-1.003$ \\
\hline Unregulated indoor climate and vehicle or cab & 13928 & 0.995 & $0.986-1.004$ & 0.996 & $0.983-1.010$ & . & . \\
\hline Indoor and outdoor work & 68519 & 1.002 & $0.998-1.006$ & 1.000 & $0.994-1.006$ &  & . \\
\hline
\end{tabular}

a Number of observations (sum of case days and referent days) included in the estimation of $\mathrm{OR}$ for these groups, subgroups and temperatures.

${ }^{\circ}$ Odds of injury for subgroups for which no evidence of a non-linear relationship between minimum temperature and injury risk was evident.

c Odds of injury for subgroups for which evidence of a curvilinear relationship between minimum temperature and injury risk was evident.

Our study has a number of strengths. Firstly, it is one of few published studies to quantify the association between high ambient temperatures and workrelated injury and one of the first to use a case-crossover approach. A strength of this design is that time-invariant individual-level confounders are controlled for by design as are time-variant confounders, such as day, month, year and season that are important in the context of the labor market. Secondly, we have used a novel classification of occupational characteristics to minimize the generalities of the ANZSCO occupation codes, which groups workers according to broad specializations rather than describing physical requirements or exposures relevant to occupational health during hot weather. For instance workers within the construction industry might be involved in outdoor, heavy labor most of the time, or could be employed in an administrative capacity in an air-conditioned indoor environment. Thirdly, to minimize exposure misclassification we have allocated meteorological exposure data to each case according to the proximity of their workplace and place of residence to the nearest of eight weather observation station. Finally we have used both daily maximum and minimum temperatures in our analyses, providing insight into the impact of warm nights on risk of injury not previously explored.

This study has a number of limitations including potential exposure misclassification, despite our efforts to minimize this. We do not know the temperature to which individuals were actually exposed; only personal temperature exposure monitors could provide this information, and this type of data collection is not feasible at the population level. In addition, use of air-conditioning will have contributed to exposure misclassification, particularly for minimum temperatures, influencing our estimates of association between ambient temperature and injury risk. The Australian Bureau of Statistics reports that $>70 \%$ of Melbourne households own some form of cooling air conditioner, and that this is unevenly distributed with air conditioner ownership being least for inner city dwellings, rental properties, and low income households $(34,35)$. Information about which workers have air conditioners and if they are used or are in working order, is not available to us. Also potentially contributing to exposure misclassification, we have assumed that workers are at their place of residence overnight, and that injuries have occurred during the day, therefore not accounting for those who work at night or are shift workers. We have not excluded personal leave days, company "rostered days off" and a summer close-down period, common in construction and building industries in Victoria. Our system of occupational classification, while validated in the Victorian context, may potentially contribute to exposure misclassification that attenuates estimated effects.

Secondly, a composite index of heat stress such as apparent temperature may have given a more accurate measure of heat stress, however we chose not to use this 
as the data available to us only permitted calculation of an approximation, and the limited popular use of this term in the Melbourne context limits its usefulness for future policy translation. Thirdly, the case-crossover design has a number of advantages over a time series analysis, but there are also limitations. This design assumes that exposures show no time trends within individual strata (14). We have attempted to minimize any trends by selecting only the hottest months for analysis. Additionally, any potential confounders acting at the individual level that arise over a short period of time (eg, state-related variables such as acute infection, co-worker absence, power outage) will not be controlled with this approach (36). Finally, the Worksafe Victoria threshold criteria for acceptance of a workers' compensation claim ( $\geq 10$ days absence from work and minimum healthcare costs) very likely limits our sampling of work-related injury cases to just the most serious injuries.

The findings from this study have important public health implications. We have identified young workers, male workers and workers engaged in the most physically demanding occupations to be vulnerable to injury during hot days. We have also found minimum overnight temperature to influence risk of injury the following day for young workers, female workers, older workers, and workers engaged in low-strength work and in climate controlled work environments. Across Australia, future global warming is likely to be experienced as an increased frequency and intensity of hot days, warm nights, and heat waves (9). This will potentially expose more workers to high ambient temperatures more often. For Melbourne, the number of days with maximum temperature exceeding $35{ }^{\circ} \mathrm{C}$ per year is expected to increase from a current average of 8 days to 16-22 days by 2070 , depending on climate scenarios (37). Average warming across Australia since 1910 has been greater for overnight minimum temperatures $\left(1.1^{\circ} \mathrm{C}\right)$ than for daytime maximum temperatures $\left(0.8^{\circ} \mathrm{C}\right)$ over the same period, and this trend is set to continue (9). In light of these climate projections, and the existing health burden of work-related injuries, it is imperative that occupational health and safety policies and guidelines acknowledge the increased risks of acute work-related injury associated with high ambient daytime and overnight temperatures and that information about vulnerable groups is incorporated in awareness raising training and educational material targeted at workers and employers.

\section{Acknowledgments}

Compensation data was provided by the Institute for Safety, Compensation and Recovery Research (ISCRR). While completing this work, Peter Smith was supported by a Discovery Early Career Researcher Award from the Australian Research Council. He is currently supported through a Research Chair in Gender, Work \& Health from the Canadian Institutes of Health Research.

\section{References}

1 International Labour Organisation. Safety and Health at Work [Internet] International Labour Office, Geneva [accessed 25 January 2016]; Available from: http://www.ilo.org/global/ topics/safety-and-health-at-work/lang--en/index.htm.

2. World Health Organisation. Protecting workers' health: Fact sheet N’389 April 2014 [accessed 8 June 2016]; Available from: http://www.who.int/mediacentre/factsheets/fs389/en/.

3. Fogleman M, Fakhrzadeh L, Bernard TE. The relationship between outdoor thermal conditions and acute injury in an aluminum smelter. Int J Ind Ergon. 2005;35(1):47-55. http:// dx.doi.org/10.1016/j.ergon.2004.08.003.

4. Morabito M, Cecchi L, Crisci A, Modesti PA, Orlandini S. Relationship between work-related accidents and hot weather conditions in Tuscany (central Italy). Ind Health. 2006;44. http://dx.doi.org/10.2486/indhealth.44.458.

5. Xiang J, Bi P, Pisaniello D, Hansen A, Sullivan T. Association between high temperature and work-related injuries in Adelaide, South Australia, 2001-2010. Occ Environ Med. 2014;71:246-52. http://dx.doi.org/10.1136/ oemed-2013-101584.

6. Adam-Poupart A, Smargiassi A, Busque M-A, Duguay P, Fournier M, Zayed J, et al. Effect of summer outdoor temperatures on work-related injuries in Quebec (Canada). Occ Environ Med. 2015;72(5):338-45. http://dx.doi. org/10.1136/oemed-2014-102428.

7. IPCC. Climate Change 2014: Synthesis Report. Contribution of Working Groups I, II and III to the Fifth Assessment Report of the Intergovernmental Panel on Climate Change [Core Writing Team, R.K. Pachauri and L.A. Meyer (eds.)]. IPCC, Geneva, Switzerland. Summary for Policy Makers, p. 2.

8. Meehl GA, Tebaldi C. More intense, more frequent, and longer lasting heat waves in the 21 st century. Science. 2004;305(5686):994-7. http://dx.doi.org/10.1126/ science.1098704.

9. Australian Government Bureau of Meteorology, CSIRO. State of the Climate 2014. [cited accessed 8 March 2016]; Available from: http://www.bom.gov.au/state-of-theclimate/documents/state-of-the-climate-2014_low-res. pdf?ref=bottom.

10. Ye X, Wolff R, Yu W, Vaneckova P, Pan X, Tong S. Ambient temperature and morbidity: a review of epidemiological evidence. Environ Health Perspect. 2012;120(1):19-28. http:// dx.doi.org/10.1289/ehp.1003198.

11. Hajat S, Kosatky T. Heat-related mortality: a review and exploration of heterogeneity. J Epidemiol Community Health. 2010;64(9):753-60. http://dx.doi.org/10.1136/ jech.2009.087999. 
12. Australian Bureau of Statistics. Australian Social Trends, Dec 2009. Cat. No. 4102.0 Canberra: ABS; 2009 [cited 5 October 2016].

13. Maclure M. The case-crossover design: a method for studying transient effects on the risk of acute events. Am J Epidemiol. 1991;133(2):144-53

14. Janes H, Sheppard L, Lumley T. Case-Crossover Analyses of Air Pollution Exposure Data: Referent Selection Strategies and Their Implications for Bias. Epidemiology. 2005;16(6):71726. http://dx.doi.org/10.1097/01.ede.0000181315.18836.9d.

15. Australian Bureau of Statistics. Regional Population Growth, Australia, 2014-15. Cat. no. 3218.0. 2016.

16. Loughnan ME, Nicholls N, Tapper NJ. When the heat is on: Threshold temperatures for AMI admissions to hospital in Melbourne Australia. Appl Geogr. 2010;30(1):63-9. http:// dx.doi.org/10.1016/j.apgeog.2009.08.003.

17. Sturman A, Tapper NJ. The weather and climate of Australia and New Zealand (2nd ed.) Melbourne, Victoria, Australia: Oxford University Press. 2006.

18. Prang K-H, Hassani-Mahmooei B, Collie A. Compensation Research Database: population-based injury data for surveillance, linkage and mining. BMC Research Notes. 2016;9(1):1-11. http://dx.doi.org/10.1186/s13104-0162255-4.

19. Ruseckaite R, Collie A. Repeat workers' compensation claims: risk factors, costs and work disability. BMC Public Health. 2011;11(1):1-8. http://dx.doi.org/10.1186/1471-2458-11492.

20. WorkSafe Victoria. Statistical Summary 2011-12. 2012 [accessed 8 June 2016]; Available from: http://www. worksafe.vic.gov.au/forms-and-publications/forms-andpublications/statistical-summary2.

21. Nicholls N, Skinner, C., Loughnan, M. and Tapper, N. A simple heat alert system for Melbourne, Australia. Int J Biometeorol. 2008;52(5):375-84. http://dx.doi.org/10.1007/s00484-0070132-5.

22. Australian Government Bureau of Meteorology. Thermal comfort observations. 2010 [accessed 8 June 2016]; Available from: http://www.bom.gov.au/info/thermal_stress/.

23. Parsons KC. Human thermal environments: The Effects of Hot, Moderate, and Cold Environments on Human Health, Comfort and Performance. 2nd ed. London, UK: Taylor \& Francis; 2003.

24. Government of Canada. Human Resources \& Skills Development Canada. National occupational classification career handbook. Ottawa, ON. 2011.

25. Smith PM, Berecki-Gisolf J. Age, occupational demands and the risk of serious work injury. Occupational Medicine -Oxford-. 2014;64(8):571-6. http://dx.doi.org/10.1093/ occmed/kqu125.
26. Smith P, Black O, Keegel T, Collie A. Are the Predictors of Work Absence Following a Work-Related Injury Similar for Musculoskeletal and Mental Health Claims? Journal of Occupational Rehabilitation. 2014;24(1):79-88. http://dx.doi. org/10.1007/s10926-013-9455-8.

27. Morabito M, Profili F, Crisci A, Francesconi P, Gensini GF, Orlandini S. Heat-related mortality in the Florentine area (Italy) before and after the exceptional 2003 heat wave in Europe: an improved public health response? International Journal of Biometeorology. 2012;56(5):801-10. http://dx.doi. org/10.1007/s00484-011-0481-y.

28. Salminen S, Oksanen T, Vahtera J, Sallinen M, Härmä M, Salo P, et al. Sleep disturbances as a predictor of occupational injuries among public sector workers. J Sleep Res. 2010;19(1p2):207-13.

29. Gabel CL, Gerberich SG. Risk Factors for Injury among Veterinarians. Epidemiology. 2002;13(1):80-6. http://dx.doi. org/10.1097/00001648-200201000-00013.

30. Tokizawa K, Sawada S-i, Tai T, Lu J, Oka T, Yasuda A, et al. Effects of partial sleep restriction and subsequent daytime napping on prolonged exertional heat strain. Occup Environ Med. 2015;72(7):521-8. http://dx.doi.org/10.1136/ oemed-2014-102548.

31. Centers for Disease Control and Prevention. Heat-related deaths--Four states, July-August 2001, and United States, 1979-1999. MMWR. 2003;52(26):610-3.

32. Pilcher JJ, Nadler E, Busch C. Effects of hot and cold temperature exposure on performance: a meta-analytic review. Ergonomics. 2002;45(10):682-98. http://dx.doi. org/10.1080/00140130210158419.

33. McInnes JA, Clapperton AJ, Day LM, MacFarlane EM, Sim MR, Smith P. Comparison of data sets for surveillance of work-related injury in Victoria, Australia. Occup Environ Med. 2014;71(11):780-7. http://dx.doi.org/10.1136/ oemed-2014-102243.

34. Australian Bureau of Statistics. 4602.2 - Household Water and Energy Use, Victoria, October 2011. Cat No 4602.2. 2012.

35. Australian Bureau of Statistics. Household Water and Energy Use, Victoria, October 2009. Cat No 4602.2. 2010.

36. Checkoway H, Pearce N, Kriebel D. Selecting appropriate study designs to address specific research questions in occupational epidemiology. Occup Environ Med. 2007;64(9):633-8. http:// dx.doi.org/10.1136/oem.2006.029967.

37. Australian Government Bureau of Meteorology and CSIRO. Climate change in Australia. 2015 [accessed 6 June 2016]; Available from: http://www.climatechangeinaustralia.gov. $\mathrm{au} / \mathrm{en} /$.

Received for publication: 6 July 2016 